\title{
CONTRIBUCIÓN NUTRICIONAL DEL PROGRAMA COMEDORES ESCOLARES A LA POBLACIÓN INFANTIL DE DIEZ ESCUELAS MUNICIPALES DE LA CIUDAD DE CÓRDOBA, ARGENTINA.
}

\section{NUTRITIONAL CONTRIBUTION THE SCHOOL CANTEENS PROGRAM TO THE CHILDREN OF TEN MUNICIPAL SCHOOLS IN THE CITY OF CÓRDOBA, ARGENTINA.}

\author{
Daniela Moyano ${ }^{1,3}$, Nilda Raquel Perovic ${ }^{2}$.
}

\section{Resumen:}

Introducción: Los comedores escolares son uno de los programas más generalizados de las últimas décadas, donde pueden tener impactos relevantes en la nutrición, salud, crecimiento y desarrollo integral en niños/as. Objetivo: Evaluar la contribución nutricional del programa Comedores Escolares (PCE) a la población infantil de escuelas municipales de Córdoba. Material y Métodos: Fue un estudio descriptivo y analítico. Se empleó una guía de observación y mediciones de pesaje de raciones en comedores escolares y recordatorios alimentarios de 24 hs. a 150 niños/as escolarizados. Resultados: Los desayunos/meriendas tuvieron déficit en energía, calcio, vitamina $\mathrm{A}$ y $\mathrm{C}$ y hierro y exceso de azúcares simples. Los almuerzos tuvieron exceso en grasas saturadas y déficit en energía, calcio, hierro, fibra y vitamina A. El consumo de alimentos en escolares fue alto en calorías dispensables, azúcares simples y grasas saturadas y deficitario en fibra, calcio y vitamina $A$, asociados a algunas características sociodemográficas. Hubo asociaciones entre la falta de adecuación de la ingesta a la meta en calcio y vitamina A y la calidad poco óptima del comedor en estos nutrientes $(p<0,05)$. Conclusiones: Se concluye que en este contexto hay aspectos nutricionales que son necesarios mejorar.

Palabras clave: niños/niñas; aportes nutricionales; comedor escolar; ingesta alimentaria-nutricional.

\section{Abstract:}

Introduction: School canteens are one of the most widespread programs in recent decades which may have significant impacts on nutrition, health, growth and development in children. Objective: To assess the nutritional contribution of School Canteens (PCE) program to children from municipal schools of Cordoba. Material and Method: Was a descriptive and analytical study. I applied an observation guide and ration weighing measurements of the school canteens food supply and food recall method was used 24 hours a sample of 170 school children. Results: The breakfasts/snacks they had a deficit in Calcium, vitamin A and $\mathrm{C}$ and iron and excess of simple sugars. The lunches they had a excess of saturated fats and deficit of energy, calcium, iron, fiber and vitamin A. The food consumption of school children was high in discretionary calories, simple sugars and saturated fats and deficit in fiber, calcium and vitamin A, associated with some sociodemographic characteristics. There had associations between the inadequacy of the target intake of calcium and vitamin found and suboptimal quality and dining in these nutrients $(p<$ $0,05)$. Conclusions: We conclude what in this context there are nutritional aspects that are necessary improve.

Keywords: children; nutritional aports; school canteen; nutritional intake.

\footnotetext{
1 Licenciada en Nutrición. Magíster en Salud Pública. Escuela de Nutrición, Facultad de Ciencias Médicas, Universidad Nacional de Córdoba, Argentina

2 Licenciada en Nutrición. Doctora en Ciencias de la Salud. Escuela de Nutrición, Facultad de Ciencias Médicas, Universidad Nacional de Córdoba, Argentina

3 Email de contacto: moyanodaniela12@gmail.com
} 


\section{Introducción}

Los programas alimentarios escolares (PAE) como también otros programas sociales con enfoque alimentario en Argentina, han experimentado una constante y marcada reconfiguración desde los años ochenta y noventa hasta la actualidad ${ }^{1}$.

Dentro de estos programas, los comedores escolares son uno de los más generalizados por las consecuencias que el déficit en la ingesta produce en este grupo etario ${ }^{2}$, tienen carácter de política focalizada y asistencial, con diferentes modalidades de gestión, donde en el país su ejecución es descentralizada desde el año 1992, tras la sanción de la Ley 24.049, orientándose a atender la población con vulnerabilidad social.

Existe evidencia de la estrecha asociación entre pobreza, malnutrición y trastorno en el crecimiento y desarrollo de niños $/ a^{3}{ }^{3}$, donde el estado de nutrición y salud es el producto de la constelación de diversos determinantes, entre ellos, culturales, sociales, económicos como así también políticos y de accesibilidad a bienes y servicios ${ }^{3}$.

Ya desde hace varios años, se viene demostrando a nivel internacional, nacional y local situaciones insatisfactorias de salud prevalentes en la población infantil caracterizadas por la presencia de múltiples inadecuaciones nutricionales que van desde baja talla, anemia y déficit de nutrientes específicos como vitamina $\mathrm{A}$ y $\mathrm{C}$, calcio, zinc y fibra, ocasionada por una escasa ingesta de frutas, verduras, granos integrales, legumbres y lácteos hasta otras problemáticas actualmente en ascenso como es el caso del consumo elevado o en exceso de alimentos ricos en energía, calorías dispensables, grasas saturadas, azúcares simples y sodio contribuyendo de manera directa a un aumento en la prevalencia de sobrepeso, obesidad y otras enfermedades crónicas no trasmisibles ${ }^{4-7}$.

Estudios internacionales y nacionales además han puesto de manifiesto inadecuaciones nutricionales en niños y niñas que realizan parte de su alimentación cotidiana en el comedor escolar, donde reflejan que las prestaciones ofrecidas no llegan a cubrir las recomendaciones para este grupo, principalmente en lo que respecta a micronutrientes (vitaminas y minerales) y en algunos casos también el aporte de energía ${ }^{2,13}$. Además ponen en evidencia que el consumo alimentario en el comedor presenta déficit en algunos grupos de alimentos, en especial el de vegetales y lácteos y que existe la necesidad de analizar las prestaciones destinadas a los niños que requieren una alimentación especial ${ }^{2,7,9,10}$.

Por otro lado, antecedentes científicos evidencian que intervenciones alimentario-nutricionales apropiadas en entornos escolares pueden ser efectivas en diferentes aspectos de la calidad de vida de la población infantil $^{7,11-14}$.

La finalidad de este trabajo es contribuir a la producción de conocimiento científico en materia de políticas públicas y brindar elementos de análisis para posibles intervenciones futuras o redefiniciones de programas alimentarios destinados a la población escolar. El objetivo principal fue analizar la contribución nutricional del programa Comedores Escolares (PCE) a la población infantil que asiste a diez escuelas municipales de la ciudad de Córdoba, en el año 2013.

\section{Materiales y Métodos}

El tipo de estudio fue descriptivo, observacional, transversal y de carácter analítico de una base de datos secundaria ${ }^{15}$ con datos recolectados durante el periodo 2013. Las unidades de análisis consistieron en 10 escuelas primarias municipales de la ciudad de Córdoba, seleccionadas por muestreo intencional según diferentes estratos socioeconómicos (tomando como variable proxy el nivel de hacinamiento) a partir del Censo Provincial de Población de Córdoba $2008^{16}$.

Dentro de las unidades de análisis se llevaron a cabo dos relevamientos de manera simultánea durante un periodo de 11 meses: de presentaciones alimentarias brindadas por el PCE y de la ingesta alimentaria de niños/as.

El relevamiento de las prestaciones alimentarias proporcionadas a través del programa Comedor Escolares (desayuno, almuerzo, merienda, refuerzo nutricional), durante dos días no consecutivos en cada comedor Almuerzo ( $n=20)$; Desayuno $(n=19)$; Merienda $(n=18)$. Se implementó un registro de observación directa y el registro de todas las prestaciones ofrecidas en el día del relevamiento detallando alimentos, bebidas, ingredientes, etc. Se incluyó en esta instancia la medición de pesada de raciones utilizando en dicho procedimiento una balanza electrónica portátil GAMA con capacidad de hasta $3 \mathrm{~kg}$; precisión $\pm 1 \mathrm{~g}$. 
El universo consistió en todos los niños/as asistentes a 10 escuelas municipales de Córdoba, donde la selección de los escolares a los que se aplicó el método recordatorio de 24 hs. $(\mathrm{R} 24 \mathrm{H})^{17}$ fue a través de un muestreo por cuotas (muestreo no aleatorio) donde no se requirió el cálculo de tamaño muestral.

Se determinó como criterios de inclusión a: niños/as de ambos sexos, de $5^{\circ}$ y $6^{\circ}$ grado (ya que el R24H está validado para niños mayores de 8 años ${ }^{17}$ ), a los que se encontraron bajo PCE y a los que asistieron al comedor escolar el día antes de realizar el relevamiento a través del R24H.

Se excluyeron a los niños/as que no presentaron consentimiento informado firmado por los padres o tutores y/o a los que tuvieron alguna dificultad cognitiva que no permitió implementar R24H.

Durante la implementación del método $\mathrm{R} 24 \mathrm{H}$ y a fin de minimizar posibles sesgos se empleó la técnica de cuatro pasos de Gibson, el uso de modelos visuales de alimentos validados, recetas estandarizadas e instrumentos caseros (cucharas, tazas u otros) ${ }^{17}$.

Para estimar la distribución de la ingesta habitual de la población bajo análisis se realizó dos observaciones una submuestra ${ }^{17}$. Por esta razón y para garantizar la factibilidad del relevamiento se determinó realizar una segunda aplicación del método $\mathrm{R} 24 \mathrm{H}$ a una submuestra del $13 \%$ de niños/as ya entrevistados en cada una de las escuelas durante un día posterior y no consecutivo a la primera entrevista. Se realizó el ajuste estadístico por medio del método "Multiple Source Method" ${ }^{17}$.

Para este estudio se tradujo la ración de alimentos de cada prestación de los comedores y de la ingesta de niños a unidades nutricionales utilizando la base de composición de alimentos del programa informático SARA $^{6}$.

\section{Variables}

Sociodemográfica: edad (años); sexo (varón o mujer); nivel de educación alcanzado (grado escolar) y turno de asistencia a la escuela (mañana o tarde).

Calidad nutricional de las prestaciones alimentarias del PCE: Adecuación del contenido de nutrientes críticos por déficit (vitaminas A y C, calcio, hierro, zinc y fibra) y por exceso (grasas saturadas, azúcares, sodio) a los criterios de calidad ${ }^{18-22}$ de las prestaciones alimentarias del comedor escolar (Por debajo de la meta/Adecuada a la meta/Por encima de la meta)

Adecuación de la ingesta de energía y nutrientes críticos por déficit y por exceso de niños y niñas que asisten al comedor escolar a las metas nutricionales ${ }^{18,19,22-27}$ (Por debajo de la meta/Adecuada a la meta/ Por encima de la meta) necesarias de alcanzar en la edad escolar.

Se realizó un análisis estadístico univariado utilizando medidas de posición y dispersión y un análisis bivariado empleando pruebas de significación estadística. Para las variables numéricas se utilizó la prueba de Shapiro-Wilk para contrastar la normalidad del conjunto de datos $(p>0,2)$ y prueba de Fisher $(p>0,05)$. Se utilizó la prueba T de Student, la prueba no paramétrica de Kruskal Wallis, la prueba del $x 2$ y el método de regresión logística simple $(p<0,05)$. El software estadístico utilizado para todos los análisis fue Infostat.

\section{Aspectos éticos}

En el estudio marco desde donde se tomó la base de datos secundaria ${ }^{15}$, antes de comenzar el relevamiento de ingesta alimentaria de niños/as se solicitó la firma del consentimiento informado a padres, tutores o representantes legales luego de haber sido exhaustivamente informados y haber comprendido la información recibida a cerca del propósito, la duración, la metodología de recolección de datos, los beneficios y la confidencialidad de la información.

\section{Resultados}

La muestra quedó constituida por un $\mathrm{N}=150$, donde el $58,7 \%$ fueron niñas y un $41,3 \%$ niños y la edad predominante de los encuestados fue entre diez y once años (79\%), un menor porcentaje tuvo edades superiores. La media de edad fue superior en el grupo de niños $(11 \pm 1,1)$ que en de las niñas $(10,9 \pm 0,9)$.

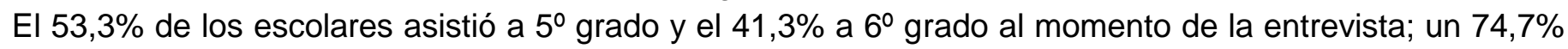
asistió a la escuela durante el turno mañana mientras que el $25,3 \%$ al turno tarde. 
En el análisis de la adecuación de la oferta de alimentos del PCE a metas nutricionales se observó que solo el $2,7 \%$ de desayunos y meriendas alcanzó la meta de energía propuesta para el grupo de niños/as; gran parte de estas prestaciones tuvieron aportes de azúcares simples por encima de la meta (solo el $5,4 \%$ se adecuó a la meta). Algunos micronutrientes esenciales fueron de calidad inadecuada. En este sentido se encontró que el total de las prestaciones no se adecuaron a los criterios propuestos en cuanto a la vitamina $\mathrm{A}$, calcio, zinc y vitamina $\mathrm{C}$ (Tabla 1 ).

Tabla 1: Adecuación de la oferta de Comedores Escolares y de ingesta de niños/as a metas nutricionales de la Ciudad de Córdoba, Argentina. 2013

\begin{tabular}{|c|c|c|}
\hline Presencia de adecuación de la oferta de comedores escolares & $\mathrm{N}$ & $\%$ \\
\hline \multicolumn{3}{|l|}{ Desayunos y Meriendas $(N=37)$} \\
\hline Vitamina A $(\mu \mathrm{g}) *$ & 0 & 0 \\
\hline Calcio $(\mathrm{mg}) *$ & 0 & 0 \\
\hline Zinc $(\mathrm{mg}) *$ & 0 & 0 \\
\hline Vitamina $\mathrm{C}(\mathrm{mg}) *$ & 0 & 0 \\
\hline Fibra $(\mathrm{g}) *$ & 2 & 5,4 \\
\hline Hierro $(\mathrm{mg}) *$ & 11 & 29,7 \\
\hline Azúcares $(\mathrm{g}) *$ & 2 & 5,4 \\
\hline Sodio $(\mathrm{mg}) *$ & 27 & 72,9 \\
\hline Grasas saturadas $(\mathrm{g}) *$ & 24 & 64,9 \\
\hline Energía $(\mathrm{Kcal}) *$ & 1 & 2,7 \\
\hline \multicolumn{3}{|l|}{ Almuerzos $(N=20)$} \\
\hline Vitamina A $(\mu \mathrm{g}) *$ & 2 & 10 \\
\hline Calcio $(\mathrm{mg}) *$ & 6 & 30 \\
\hline Zinc $(\mathrm{mg}) *$ & 14 & 70 \\
\hline Vitamina $\mathrm{C}(\mathrm{mg}) *$ & 14 & 70 \\
\hline Fibra $(\mathrm{g}) *$ & 1 & 5 \\
\hline Hierro $(\mathrm{mg}) *$ & 14 & 70 \\
\hline Azúcares simples $(\mathrm{g}) *$ & 18 & 90 \\
\hline Sodio $(\mathrm{mg}) *$ & 20 & 100 \\
\hline Grasas saturadas $(\mathrm{g}) *$ & 11 & 55 \\
\hline Energía $(\mathrm{Kcal}) *$ & 0 & 0 \\
\hline \multicolumn{3}{|l|}{ Presencia de adecuación de la ingesta de niños/as $(n=170)$} \\
\hline Vitamina $\mathrm{A}(\mu \mathrm{g}) *$ & 35 & 20,6 \\
\hline Calcio (mg) * & 6 & 3,5 \\
\hline Zinc $(\mathrm{mg}) *$ & 136 & 80 \\
\hline Vitamina $\mathrm{C}(\mathrm{mg}) *$ & 114 & 67 \\
\hline Fibra $(\mathrm{g}) *$ & 1 & 0,6 \\
\hline Hierro $(\mathrm{mg}) *$ & 162 & 95,2 \\
\hline Azúcares simples (g) * & 85 & 50 \\
\hline Sodio $(\mathrm{mg}) *$ & 111 & 65 \\
\hline Grasas saturadas $(\mathrm{g}) *$ & 37 & 22 \\
\hline Energía $(\mathrm{Kcal}) *$ & 29 & 17 \\
\hline Calorías dispensables (Kcal)* & 32 & 19 \\
\hline
\end{tabular}

En los almuerzos analizados se observó una buena calidad en nutrientes críticos por excesos principalmente en lo que respecta a azúcares y sodio (90\% y $100 \%$ respectivamente se adecuaron a los criterios de calidad); cabe aclarar en este punto que en el análisis de sodio no consideró el aporte a través de la sal agregada, solo se tuvo en cuenta el aportado a través de los alimentos. Por otra parte el aporte de energía y de calcio de los almuerzos evaluados no alcanzaron las metas establecidas en el $100 \%$ de las prestaciones. Una alta proporción de almuerzos tuvieron además una calidad poco óptima en el aporte de hierro y de fibra dietética (Tabla 1). 
A partir de evaluar la adecuación (\%) a las metas nutricionales de la ingesta de energía y nutrientes críticos por déficit y exceso de niños y niñas que asistieron al comedor escolar durante el año 2013, se pudo observar que gran parte de ellos tuvieron una ingesta de calorías dispensables por encima de las recomendaciones (solo el 19\% adecuó la ingesta a la meta); esto se visualizó de igual manera en torno a las grasas saturadas (22\% alcanzó la meta) y energía total (el $17 \%$ adecuó la ingesta a la meta) y una alta proporción de niños/as no alcanzó la meta de consumo de fibra, calcio y vitamina A (Tabla 1).

Al analizar las medias de ingesta de energía y nutrientes críticos por déficit y exceso (como grasas saturadas, calorías dispensables, vitamina A, vitamina $\mathrm{C}$, zinc y fibra) del total de observaciones alimentarias realizadas a niños/as según el turno de asistencia a la escuela (variable sociodemográfica), se encontraron diferencias estadísticamente significativas (valores de $p<0,05$ y un IC del 95\%) siendo estos valores superiores en el grupo de escolares que asistieron a la escuela en el turno mañana con respecto a los del turno tarde.

A partir del modelo de regresión logística aplicado a las variables de adecuación a las metas nutricionales y considerando como co-variables de ajuste a las sociodemográficas (sexo, turno de asistencia escolar, edad y nivel de educación alcanzado) se encontró que en el alcance de las metas nutricionales en todos los nutrientes bajo análisis uno de los principales factores de riesgo fue el turno tarde de asistencia escolar, siendo el efecto más marcado en la meta de hierro $(\mathrm{OR}=6,22 \mathrm{IC}=1,39-27,82)$ y de vitamina $\mathrm{A}$ $(\mathrm{OR}=4,28 \mathrm{IC}=1,20-15,29)$. Mientras que el sexo masculino apareció como un factor de protección frente al alcance de las recomendaciones nutricionales, principalmente en energía $(O R=0,32 \mathrm{IC}=0,14-0,74)$, vitamina $A(O R=0,33 \mathrm{IC}=0,15-0,93)$ y zinc $(\mathrm{OR}=0,24 \mathrm{IC}=0,09-0,63)($ Tabla 2$)$.

Tabla 2: Relación entre adecuación de la ingesta de niños/as a la meta nutricional y características sociodemográficas de la Ciudad de Córdoba,

Argentina. 2013

\begin{tabular}{|c|c|c|}
\hline Ingesta $(n=170)$ & $\begin{array}{c}\text { OR } \\
(\text { IC 95\%) }\end{array}$ & $\mathbf{p}$ \\
\hline Energía & ref. & \\
\hline Género (Masculino) & $0,32(0,14-0,74)$ & $0,008 *$ \\
\hline Edad (10 años) & $0,15(0,02-1,25)$ & 0,07 \\
\hline Turno (Tarde) & $1,49(0,51-4,35)$ & 0,47 \\
\hline Grado $\left(6^{\circ}\right)$ & $0,26(0,03-2,19)$ & 0,21 \\
\hline Sodio & ref. & \\
\hline Género (Masculino) & $1,71(0,89-3,31)$ & 0,10 \\
\hline Edad (10 años) & $0,47(0,17-1,28)$ & 0,14 \\
\hline Turno (Tarde) & $0,37(0,15-0,87)$ & $\mathbf{0 , 0 2} *$ \\
\hline Grado $\left(6^{\circ}\right)$ & $0,37(0,15-0,87)$ & $0,02 *$ \\
\hline Grasas saturadas & ref. & \\
\hline Género (Masculino) & $1,31((0,69-2,49)$ & 0,41 \\
\hline Edad (10 años) & $0,32(0,12-0,87)$ & $\mathbf{0 , 0 2} *$ \\
\hline Turno (Tarde) & $1,47(0,7-3,11)$ & 0,30 \\
\hline Grado $\left(6^{\circ}\right)$ & $1,04(0,4-2,72)$ & 0,93 \\
\hline Hierro & ref. & \\
\hline Género (Masculino) & $0,47(0,09-2,52)$ & 0,38 \\
\hline Edad (10 años) & $0,81(0,07-9,96)$ & 0,87 \\
\hline Turno (Tarde) & $6,22(1,39-27,82)$ & $0,01 *$ \\
\hline Grado $\left(6^{\circ}\right)$ & $1,97(0,2-19,4)$ & 0,56 \\
\hline Zinc & ref. & \\
\hline Género (Masculino) & $0,24(0,09-0,63)$ & $0,003 *$ \\
\hline Edad (10 años) & $2,39(0,47-12,19)$ & 0,29 \\
\hline Turno (Tarde) & $2,36(0,99-5,65)$ & $0,05 *$ \\
\hline Grado $\left(6^{\circ}\right)$ & $5,05(1,03-24,71)$ & $0,04 *$ \\
\hline Vitamina A & ref. & \\
\hline Género (Masculino) & $0,33(0,15-0,93)$ & $0,006 *$ \\
\hline Edad (10 años) & $0,9(0,22-3,72)$ & 0,88 \\
\hline
\end{tabular}




\begin{tabular}{lcc} 
Turno (Tarde) & $4,28(1,20-15,29)$ & $\mathbf{0 , 0 2}$ \\
Grado $\left(6^{\circ}\right)$ & $0,43(0,11-1,68)$ & 0,22 \\
\hline Vitamina C & ref. & \\
\hline Género (Masculino) & $1,41(0,72-2,78)$ & 0,31 \\
Edad (10 años) & $0,8(0,27-2,33)$ & 0,68 \\
Turno (Tarde) & $2,72(1,29-5,75)$ & $\mathbf{0 , 0 0 8 *}$ \\
Grado $\left(6^{\circ}\right)$ & $1,73(0,62-4,82)$ & 0,29 \\
\hline & OR=Odds Ratio*Diferencias estadísticamente significativas con un valor $\mathbf{p}<0,05$. &
\end{tabular}

A partir del análisis bivariado se constató que el 100\% en niños/as que asistió al turno tarde realizó el almuerzo solamente en la escuela, mientras que se encontró que mas de la mitad de los que asistieron al turno mañana almorzaron tanto en la escuela como en el hogar, estas diferencias fueron estadísticamente significativas ( $p<0,05$ y IC 95\%). Además se constató asociaciones entre la adecuación de la ingesta de niños/as a las metas propuestas y la calidad del comedor. Por ejemplo, se observó que el 96\% de los escolares que no adecuaron su ingesta a las metas nutricionales de calcio asistió a un comedor escolar que tenía una baja calidad en este mismo micronutriente $(p=0,0001$ y IC=95\%), esta situación se presentó de manera similar en torno a la vitamina $A(p=0,0001$ y $I C=95 \%)($ Tabla 3$)$.

Tabla 3: Relación entre calidad nutricional de comedores escolares y la adecuación a metas nutricionales de niños/as de la Ciudad de Córdoba, Argentina. 2013

\begin{tabular}{|c|c|c|c|}
\hline Calidad nutricional del comedor & $\begin{array}{c}\text { Adecuación a la meta } \\
\qquad \mathrm{N}(\%)\end{array}$ & $\begin{array}{c}\text { No adecuación a la meta } \\
\qquad \mathrm{N}(\%)\end{array}$ & $\mathrm{p}$ \\
\hline \multicolumn{4}{|l|}{ Grasas saturadas } \\
\hline Adecuada & $19(22)$ & $66(78)$ & 0,9 \\
\hline No adecuada & $19(22)$ & $66(78)$ & \\
\hline \multicolumn{4}{|l|}{ Sodio } \\
\hline Adecuada & $111(0)$ & $59(100)$ & 0.0001* \\
\hline No adecuada & $0(0)$ & $0(0)$ & \\
\hline \multicolumn{4}{|l|}{ Azúcares } \\
\hline Adecuada & $7(41)$ & $10(59)$ & 0,44 \\
\hline No adecuada & $78(51)$ & $75(49)$ & \\
\hline \multicolumn{4}{|l|}{ Fibra } \\
\hline Adecuada & $0(0)$ & $17(10)$ & 0,74 \\
\hline No adecuada & $1(1)$ & $152(90)$ & \\
\hline \multicolumn{4}{|l|}{ Hierro } \\
\hline Adecuada & $146(90)$ & $7(88)$ & 0,8 \\
\hline No adecuada & $16(10)$ & $1(12)$ & \\
\hline \multicolumn{4}{|l|}{ Calcio } \\
\hline Adecuada & $0(0)$ & $0(0)$ & 0.0001* \\
\hline No adecuada & $6(4)$ & $164(96)$ & \\
\hline \multicolumn{4}{|l|}{ Zinc } \\
\hline Adecuada & $80(59)$ & $22(65)$ & 0,5 \\
\hline No adecuada & $56(41)$ & $12(35)$ & \\
\hline \multicolumn{4}{|l|}{ Vitamina A } \\
\hline Adecuada & $0(0)$ & $0(0)$ & $0.0001 *$ \\
\hline No adecuada & $35(21)$ & $135(79)$ & \\
\hline \multicolumn{4}{|l|}{ Vitamina C } \\
\hline Adecuada & $83(73)$ & $36(64)$ & 0,25 \\
\hline No adecuada & $31(27)$ & $20(36)$ & \\
\hline
\end{tabular}




\section{Discusión}

Las políticas alimentarias en Argentina entre éstas los comedores escolares (CE), surgieron durante la década del `80, luego fueron profundizadas en el periodo de convertibilidad en los `90 y resurgieron a partir de la crisis 2001 donde se fueron consolidando, descentralizado, institucionalizado y legitimado durante los sucesivos gobiernos democráticos; sin embargo aún hoy y en base a la evidencia disponible, presentan múltiples desafíos, entre estos el de garantizar condiciones óptimas y de calidad de los servicios durante los procesos de gestión y, por otra parte, poder alcanzar objetivos nutricionales y sanitarios favorables en la población infantil ${ }^{2,7,8-10}$.

La realidad nutricional y de salud de niños y niñas hoy es uno de los problemas más complejos a nivel social y sanitario que deben afrontar las sociedades de los países de Latinoamérica; esta realidad se caracteriza por fenómenos caracterizados por una "doble carga de malnutrición" ${ }^{4,6}$ o como lo expresa Aguirre (2011) el "hambre oculto" ${ }^{28}$, siendo producto de la interacciones de múltiples determinantes y condicionantes sociales y de sucesivas décadas de vulnerabilidad, pobreza y derechos no garantizados.

La doble carga de malnutrición en los primeros años de vida se encuentra presente a nivel internacional, nacional y local; expresándose en indicadores poco adecuados como es la baja talla (desnutrición crónica), la prevalencia de anemia y déficit de nutrientes críticos en niños/as como vitamina A y C, calcio, zinc y fibra, todas condicionadas por una escasa ingesta de frutas, verduras, granos integrales, legumbres y lácteos; sin embargo éstas situaciones de "déficit" coexisten con otras problemáticas actualmente prevalentes y en ascenso como es el caso del consumo elevado de alimentos ricos en energía, grasas saturadas, azúcares simples y sodio que van contribuyendo de manera directa a un aumento en la prevalencia de sobrepeso, obesidad y otras enfermedades crónicas no trasmisibles ${ }^{4-7}$.

Este escenario nutricional y epidemiológico es concordante con los hallazgos en ésta investigación donde se observó que una parte importante de la muestra de escolares presentó un consumo alimentario a base de pan, galletas y panificados dulces como facturas, tortas, etc. y donde las legumbres solo se observaron presentes en la ingesta de una pequeña parte de niños/as, ambas dentro del grupo de cereales y derivados. Al analizar específicamente el consumo de nutrientes se observó que si bien la ingesta media de energía fue acorde con las recomendaciones según sexo y edad, las inadecuaciones nutricionales por déficit y por excesos son prevalentes y preocupantes en la población bajo análisis.

Además, dentro de los procesos alimentarios y nutricionales no se pudo dejar de considerar diferentes determinantes sociales vinculados a la malnutrición y seguridad alimentaria en la infancia. En nuestro análisis se consideraron algunas variables sociales y de género. Desde esta perspectiva por ejemplo, se ha visualizado que existió una asociación marcada entre el hábito de realización del almuerzo y el turno de asistencia escolar donde el total de escolares que asistió al turno tarde realizaron el almuerzo solamente en la escuela a diferencia del turno mañana (tanto en hogar y escuela) o donde el consumo de casi todos los nutrientes fue superior en el segundo grupo vs. el primero. Estos datos fueron llamativos ya que estarían indicando un consumo diferenciado según el turno de asistencia a la escuela. Se observó además que la ingesta de algunos micronutrientes como el hierro y el zinc fueron estadísticamente mayores en el grupo de niños que en el de niñas, indicando diferencias de género y donde esta variable puede constituirse en un factor de riesgo/protección al momento de alcanzar las metas en la infancia.

Según Aguirre (2011) estos escenarios nutricionales complejos a nivel del país se vieron además plasmados a través de las prácticas estatales desde el surgimiento de políticas alimentarias de asistencia en el marco de comedores, fuertemente profundizadas durante la década neoliberal de los ` 90 , abordando la carencia desde la lógica estatal con un empobrecimiento constante de las políticas alimentarias ${ }^{29}$; hecho que además se van reproduciendo en las sucesivas décadas desde las estrategias domesticas de consumo hacia dentro de las unidades domésticas, reforzadas aún más por el mercado y la publicidad ${ }^{29}$. Estudios internacionales, nacionales y locales recientes pudieron constatar que las prestaciones alimentarias ofrecidas por comedores escolares no llegaron a cubrir las recomendaciones para el grupo en edad escolar, principalmente en lo que respecta a micronutrientes (vitaminas y minerales) y en algunos casos también el aporte de energía ${ }^{2,7-10}$. Este escenario de carencias en el marco del comedor escolar acompañado de excesos se correspondió con los resultados encontrados en éste estudio donde se detectó una baja frecuencia de oferta de algunos alimentos saludables como frutas y verduras y donde además se constató que desayunos/meriendas fueron las prestaciones que evidenciaron una baja calidad 
nutricional en energía y los nutrientes críticos esenciales como calcio, vit. A y $\mathrm{C}$ y hierro no alcanzaron la meta nutricional saludable en la infancia coexistiendo con excesos nutricionales ${ }^{4,6}$ principalmente en azúcares simples. Los almuerzos analizados fueron los que evidenciaron menores inadecuaciones en nutrientes críticos, sin embargo se determinó que una proporción importante de éstos tuvo un aporte poco adecuado en grasas saturadas.

Este trabajo reflejó, en parte, lo que vienen planteando algunos autores que ponen de manifiesto que la asistencia estatal se caracteriza por ofrecer un acceso a alimentos poco saludables y dietas desbalanceadas, influyendo de manera directa a la doble carga de malnutrición en la infancia principalmente en los grupos poblacionales más vulnerables destinatarios de estas políticas ${ }^{28,30}$, esta premisa se sustenta a partir de los hallazgos sobre las asociaciones estadísticamente significativas entre la falta de adecuación de la ingesta de escolares a la meta en el calcio y vitamina A y un aporte bajo por parte del comedor en estos nutrientes.

Se puede concluir que hay múltiples necesidades y frentes para avanzar hacia nuevos paradigmas en la implementación y gestión de la política pública de comedores escolares en pos de la salud integral de niños y niñas.

\section{Agradecimientos}

A todos los actores de las escuelas municipales de la ciudad de Córdoba, Argentina, principalmente a los niños y niñas que formaron parte del estudio marco.

\section{Bibliografía}

1. Santarsiero LH. Las políticas sociales en el caso de la satisfacción de necesidades alimentarias: Algunos elementos conceptuales para su determinación. Rev Trab Soc. [Internet]. 2012 [citado 2 dic 2013]; 159-176. Disponible <http://www.scielo.org.ar/scielo.php?script=sci_arttext\&pid=S151468712012000100010\&Ing=es\&nrm=iso >. ISSN 1514-6871.

2. Buamden S, Graciano A, Manzano G, Zummer, E. Proyecto: Encuesta a los Servicios Alimentarios de Comedores Escolares Estatales (PESCE): alcance de las metas nutricionales de las prestaciones alimentarias de los comedores escolares de Gran Buenos Aires, Argentina. Diaeta [Internet]. ene./mar. 2010. [citado 26 Ene 2013]. 28(130): [aprox. 1 p.]. Disponible en: http://www.scielo.org.ar/scielo.php?pid=S185273372010000100005\&script $=$ sci_arttext

3. Labadié S, Fernández AR, Aguirre P. Condicionantes epidemiológicos y representaciones sociales de la desnutrición infantil en la comunidad Mocoví. [Tesis de Maestría]. Córdoba: Escuela de Salud Pública. FCM. UNC; 2010.

4. Centro de Estudios Sobre Nutrición Infantil (CESNI). 1era. Jornada de Obesidad Infantil. Hacia el mapa de la obesidad en Argentina. Argentina: CESNI; 2012.

5. Mamondi V, Lavin J, Bruzzone F, Gonzalez E, Berra S. Percepción del bienestar físico en niños obesos y sedentarios, Ciudad de Córdoba, Argentina. Revista de Salud Pública. 2012; 169:48

6. Encuesta Nacional de Nutrición y Salud. Ministerio de Salud de la Nación. Buenos Aires, Argentina, 20042005. [Disponible en:http://msal.gov.ar/htm/Site/ennys/download/Implementaci\%C3\%B3n.pdf] [Acceso 30 de Ene 2013].

7. Piaggio L, Concilio C, Rolón M, Dupraz S. Alimentación infantil en el ámbito escolar: entre patios, aulas y comedores. Salud Colectiva. [Internet]. 2011; [citado 20 Mar 2013] 7 (2):199-213 [aprox. 1 p.]. Disponible en: http://www.scielo.org.ar/scielo.php?script=sci_arttext\&pid=S185182652011000200012\&lng=s

8. Bracamonte S, Carranza C, Olivero I, Laquis M. Calidad nutricional y consumo de los menúes del comedor escolar y estado nutricional de los alumnos del nivel primario de la escuela Jorge Peyrano, Ciudad de La Calera, Provincia de Córdoba, 2012. [T.I.L] Córdoba: Escuela de Nutrición. FCM. UNC; 2012.

9. Martinez Alvarez JR, Garcia Alcon R, Villarino Marin A, Serrano Morago L, Marrodan Serrano MD. Encuesta nacional sobre comedores escolares y demanda de dietas especiales. Nutr Hosp. [Internet]. 2012; [citado 20 $\begin{array}{lllll}\text { May 2013]; 27(1):252-255. } & \text { [aprox. } & 1 & \text { p.]. } & \text { Disponible }\end{array}$ en:http://scielo.isciii.es/scielo.php?pid=S021216112012000100032\&script=sci_arttext\&t/ng=es

10. Zulueta B, Xarles Irastorza I, Oliver P, Garcia Z, Victoria JC. Perfil nutricional de los menús e ingesta dietética en comedores escolares de Vizcaya. Nutr Hosp. [Internet]. 2011; [citado 5 Jun 2013]; 26(5):1183-1187. [aprox. 5 p.]. Disponible en: http://www.nutricionhospitalaria.com/pdf/5161.pdf

11. Food and Agriculture Organization of the United Nations. School Feeding and the Possibilities for Direct Purchases from Family Farming - Case Studies in Eight Countries. Santiago de Chile: FAO; 2013.

12. Cunningham-Sabo L, Lohse B. Cooking with kids positively affects fourth graders' vegetable preferences and attitudes and self-efficacy for food and cooking. Child Obes. [Internet]. 2013; [citado 14 dic 2013]; 9(6):549-56 [aprox. 1 p.]. Disponible en: http://www.ncbi.nlm.nih.gov/pubmed/24320723 
13. Fretes G, Salinas J, Vio F. Effect of a nutrition education intervention on consumption of fruits, vegetables and fish in families of prescholers and scholers. J Sch Health [Internet]. 2005; [citado 14 dic 2013]; 75(6):199-213. [aprox. 1 p.]. Disponible en: http://www.ncbi.nlm.nih.gov/pubmed/24167956

14. Food and Agriculture Organization, Brazilian Cooperation Agency. Strengthening School Meal Programmes under the Hunger Free Latin America and the Caribbean 2025 Initiative. Brazil: FAO/ABC; 2008.

15. Britos S, Virgolini M, Saraví A, Chichizola N, Moyano D, Perez L, Arroyo S. Analysis of nutrition within the school environment. Anuario 2013 Becas de Investigación "Ramón Carrillo-Arturo Oñativia". 2013.

16. Gobierno de la provincia de Córdoba. Censo Provincial de Población 2008. Disponible en: http://estadistica.cba.gov.ar/Poblaci\%C3\%B3n/Censo2008/tabid/462/language/es-AR/Default.aspx. Consultado: 30 de marzo 2014

17. Ferrari, MA. Estimación de la Ingesta por Recordatorio de 24 Horas. Diaeta, [Internet]. 2013; [citado 30 nov. 2013]; 20-25 [aprox. 1 p.]. Disponible en: <http://www.scielo.org.ar/scielo.php?script=sci_arttext\&pid=S1852$73372013000200004 \&$ lng $=e s \& n r m=i s o>$.

18. World Health Organization. Food and Agriculture Organization. Expert Consultation Report on Human Energy Requirements. Interin Report. Rome; 2001.

19. Department of Agriculture and U.S. Department of Health and Human Services. Dietary Guidelines for Americans, 2010. 7th Edition, Washington, DC: U.S. Government Printing Ofice, Diciembre 2010.

20. World Health Organization. Food and Agriculture Organization. Consultation Report on Human Vitamin and Mineral Requirements. Bangkok: WHO/FAO; 2001.

21. World Health Organization/ Food and Agriculture Organization of the United Nations. Diet, nutrition and the prevention of chronic diseases. Geneva: WHO/FAO; 2003.

22. Institute of Medicine, Food and Nutrition Board. Dietary Reference Intakes for Energy, Carbohydrat, Fiber, Fat, Fatty Acids, Cholesterol, Protein and Amino Acids. Prepublication Copy. Washington D.C: National Academy Press; 2002/2005.

23. Institute of Medicine, Food and Nutrition Board. Institute of Medicine, Food and Nutrition Board. Dietary Reference Intakes for Vitamin A, Vitamin K, Arsenic, Boron, Chromium, Copper, lodine, Iron, Manganese, Molybdenum, Nickel, Silicon, Vanadium, and Zinc. Washington D.C: National Academy Press; 2001.

24. Institute of Medicine, Food and Nutrition Board. Dietary Reference Intakes for Thiamin, Riboflavin, Niacin, Vitamin B6, Folate, Vitamin B12, Pantothenic Acid, Biotin, and Choline. Washington D.C: National Academy Press; 1998.

25. Institute of Medicine, Food and Nutrition Board. Institute of Medicine, Food and Nutrition Board. Dietary Reference Intakes for Calcium and Vitamin D. Washington D.C: National Academy Press; 2011.

26. Institute of Medicine, Food and Nutrition Board. Institute of Medicine, Food and Nutrition Board. Dietary Reference Intakes for Vitamin C, Vitamin E, Selenium, and Carotenoids. Washington D.C: National Academy Press; 2000.

27. Sociedad Argentina de Pediatría. Comité Nacional de Crecimiento y Desarrollo. Guías para la Evaluación del Crecimiento. 2da. Edición. 2001.

28. Aguirre P. Reflexiones sobre las nuevas formas del hambre en el siglo XXI: La obesidad de la Escasez. Boletín Científico Sapiens. [Internet]. 2011. [citado 20 Ene 2016]; 1:2 60-4, Disponible en: www/l ssuu.com/ sapiens_research_vol 1.

29. Aguirre P. Consecuencias del ajuste en la alimentación. Rev Voces del Fénix. [Internet]. 2011. [citado 20 Ene 2016]; Disponible en: http://www.vocesenelfenix.com/sites/default/files/numero_pdf/Voces\%20N.7\%20\%20final\%20baja_0.pdf

30. Del Castillo SE. La situación nutricional de la niñez en Latinoamérica: entre la deficiencia y el exceso, de brecha nutricional a deuda social. Rev Biomédica. [Internet]. 2012. [citado 20 Ene 2016]; 32 (4): 471-473. Disponible en: $h$ ttp://www. redalyc.org/articulo.oa?id=84324951001 